\title{
VARIACC̃̃O DOS MÓDULOS DE ELASTICIDADE E RUPTURA EM MADEIRA DE CEDRO-AUSTRALIANO POR MEIO DE ENSAIOS NÃO DESTRUTIVO E DESTRUTIVO
}

\author{
VARIATION OF ELASTICITY AND RUPTURE MODULES IN AUSTRALIAN CEDAR WOOD \\ BY NONDESTRUCTIVE AND DESTRUCTIVE TESTS
}

\begin{abstract}
Pedro Gutemberg de Alcântara Segundinho ${ }^{1}$ Adair José Regazzi ${ }^{2}$ Franco Simões Poletti ${ }^{3}$ Marcos Oliveira de Paula ${ }^{4}$ Adriano Ribeiro de Mendonça ${ }^{5}$ Fabricio Gomes Gonçalves ${ }^{5}$
\end{abstract}

\begin{abstract}
RESUMO
O módulo de elasticidade é uma propriedade mecânica importante no dimensionamento de projetos de estruturas de madeira, logo, a obtenção por meio de diversos métodos necessita ser analisada. Diante disso, este trabalho teve como objetivo avaliar a utilização de técnicas não destrutiva e destrutiva para determinar o módulo de elasticidade longitudinal aparente, levando em conta a relação vão/altura $(L / h)$, e verificar a influência do cisalhamento no módulo de elasticidade, por meio da comparação de ensaios não destrutivos com ensaios de flexão estática. A madeira utilizada no trabalho foi de Toona ciliata M. Roemer (cedroaustraliano). Os ensaios não destrutivos foram realizados por meio das técnicas de vibração transversal livre e longitudinal. Concluiu-se que o ensaio de vibração longitudinal é o método mais adequado para obtenção do módulo de elasticidade longitudinal aparente $\left(M O E_{a, l}\right)$, pois os esforços cortantes que ocorrem devido às tensões de cisalhamento não influenciaram significativamente na obtenção do $\left(M O E_{a, l}\right)$, considerando a variação da relação $L / h$. Também foi possível concluir que, a relação $L / h$, atualmente igual a 21 na ABNT NBR 7190 (1997), pode ser reduzida para 20, quando da determinação dos módulos de elasticidade longitudinal aparente e módulo de ruptura por meio do ensaio de flexão estática e vibração transversal livre. Palavras-chave: vibração longitudinal; vibração transversal livre; flexão estática.
\end{abstract}

\section{ABSTRACT}

The elastic of modulus is an important mechanical property in the design of timber structures, so obtaining it through several methods needs to be evaluated. This study aimed to evaluate the use of an efficient technique for determining the apparent longitudinal modulus taking into account the relationship range/ height $(\mathrm{L} / \mathrm{h})$ and the influence of the shear modulus by comparing non-destructive tests with static bending tests. The wood used in paper was Toona ciliata M. Roemer (Australian cedar). Non-destructive tests were performed with the techniques of longitudinal and transverse vibration. It was concluded that the assay of longitudinal vibration is most suitable method to obtain the apparent longitudinal elasticity modulus, because the shear forces that occur due to the shear stresses are not significantly influenced by the variations of the ratio $\mathrm{L} / \mathrm{h}$. It was also possible to conclude that the relationship range/height, currently 21 in ABNT's

1 Engenheiro Civil, Dr., Professor do Departamento de Ciências Florestais e da Madeira, Centro de Ciências Agrárias e Engenharias, Universidade Federal do Espírito Santo, Campus de Alegre, Av. Gov. Lindemberg, 316, CEP 29550000, Centro, Jerônimo Monteiro (ES), Brasil.p_gutemberg2001@yahoo.com.br

2 Engenheiro Agrônomo, Dr., Pesquisador do Departamento de Ciências Florestais e da Madeira, Centro de Ciências Agrárias e Engenharias, Universidade Federal do Espírito Santo, Campus de Alegre, Av. Gov. Lindemberg, 316, CEP 29550-000, Centro, Jerônimo Monteiro (ES), Brasil. adairreg@ufv.br

3 Engenheiro Industrial Madeireiro, Departamento de Ciências Florestais e da Madeira, Centro de Ciências Agrárias e Engenharias, Universidade Federal do Espírito Santo, Campus de Alegre, Av. Gov. Lindemberg, 316, CEP 29550-000, Centro, Jerônimo Monteiro (ES), Brasil. franco.s.poletti@gmail.com

4 Engenheiro Civil, Dr., Professor do Departamento de Engenharia Florestal, Universidade Federal de Viçosa, Centro de Ciências Agrárias, Av. Peter Henry Rolfs, s/n, CEP 36570-000, Viçosa (MG), Brasil. modep@ufv.br

5 Engenheiro Florestal, Dr., Professor do Departamento de Ciências Florestais e da Madeira, Centro de Ciências Agrárias e Engenharias, Universidade Federal do Espírito Santo, Campus de Alegre, Av. Gov. Lindemberg, 316, CEP 29550-000, Centro, Jerônimo Monteiro (ES), Brasil. fabricio.goncalves@ufes.br / adriano.mendonca@ufes.br

Recebido para publicação em 12/11/2014 e aceito em 19/07/2017

Ci. Fl., v. 28, n. 3, jul. - set., 2018 
standards, may be replaced by 20 , when determining apparent longitudinal elasticity modules and modulus of rupture, through the static bending and free transverse vibration tests.

Keywords: longitudinal vibration; transverse vibration; bending test.

\section{INTRODUÇÃ̃O}

A madeira sempre foi um dos materiais de construção civil mais empregado pelo ser humano. As características físicas e mecânicas e relativa abundância desse material, possibilitam sua aplicação em usos que vão desde os acabamentos de interiores às grandes estruturas, como pavilhões, coberturas e pontes. A madeira apresenta vantagens ambientais quando comparada com outros materiais de construção (cimento, concreto, aço e alumínio), por ser reciclável, renovável, biodegradável e requerer baixa necessidade de energia para seu processamento (MARQUES, 2008). Pode-se elucidar o emprego bemsucedido desse material para fins estruturais pelo fato de que em países desenvolvidos, como Noruega, Suécia, Canadá e Austrália, aproximadamente $90 \%$ das construções habitacionais são em madeira. Assim, como acontece com todos os materiais empregados na construção civil, a madeira para uso estrutural deve ser classificada pelas suas propriedades físicas e mecânicas (SEGUNDINHO et al., 2012). A Norma Brasileira Regulamentadora - NBR 7190, da Associação Brasileira de Normas Técnicas - ABNT (1997), estabelece o uso da madeira pelo sistema de classes de resistência, isto é, a norma prescreve possíveis classes de resistência ao engenheiro, atribuindo, a cada uma delas, sua resistência característica, módulo de elasticidade e densidades. No projeto de revisão da norma ABNT NBR 7190 (1997), que se encontra em análise desde 2011, sugere-se a inclusão de regras de classificação visual para distribuição da madeira em lotes de uso estrutural, conforme sugerido por Carreira e Dias (2005), a partir da qual poderá ser feita a classificação mecânica. Dessa forma o processo se inicia por meio de métodos não destrutivos, nos quais é feita uma inspeção visual, a fim de examinar algumas características intrínsecas (inclinação das fibras, nós, empenamento, anéis de crescimento, encurvamento, racha anelar e fenda), agrupando-as em lotes menos heterogêneos (FIORELLI; DIAS; COIADO, 2009) e, em seguida, faz-se a classificação mecânica da madeira com equipamentos capazes de quantificar, adequadamente, o módulo de elasticidade das peças.

Quando se deseja avaliar a resistência mecânica de peças estruturais de madeira deve-se ter em mente que há diversas formas para que isto seja concretizado. Targa, Ballarin e Biaggioni (2005), Segundinho et al. (2012) e Carreira et al. (2012) citam algumas técnicas não destrutivas, isto é, aquelas em que não há destruição das peças de madeira, como o ultrassom, a vibração transversal livre ou free-free bar (barra livre-livre), a vibração longitudinal, o raio X e o stress wave, ou ondas de tensão. Tais métodos não destrutivos são os mais empregados na determinação das propriedades físicas e mecânicas da madeira, pois são capazes de oferecer rápidas respostas e correlações lineares elevadas, tendo custos de equipamentos baixos, além de estarem se tornando cada vez mais relevantes.

Na norma ABNT NBR 7190 (1997), não está definida a obtenção do módulo de elasticidade por meio de ensaios não destrutivos, como os métodos de vibração longitudinal e transversal. Atualmente, recomenda-se uma relação $\mathrm{L} / \mathrm{h}$ maior ou igual a 21 para ensaios destrutivos (flexão estática), para que não ocorra influência do cisalhamento. Para os ensaios não destrutivos, a relação $\mathrm{L} / \mathrm{h}$ ainda não está definida. A definição normativa da relação $\mathrm{L} / \mathrm{h}$ para os métodos não destrutivos facilitará a obtenção do módulo de elasticidade por meio dessas técnicas, pois a norma poderá ser consultada e a relação $\mathrm{L} / \mathrm{h}$ estará definida para caracterização da madeira por meio destes métodos. Dessa forma, espera-se que o desenvolvimento dessa pesquisa seja de grande utilidade para as empresas e a comunidade científica, pois influenciará os métodos de cálculo de projetos de estrutura de madeira.

Este trabalho teve como objetivo comparar os valores dos módulos de elasticidade longitudinal aparente obtido por meio das técnicas não destrutiva e destrutiva, e também avaliar a influência do cisalhamento no módulo de elasticidade longitudinal aparente, obtidos pelas mesmas técnicas, levando-se em conta a relação $\mathrm{L} / \mathrm{h}$. 


\section{MATERIAL E MÉTODO}

A madeira utilizada no presente estudo foi proveniente de 8 árvores de Toona ciliata M. Roemer (cedro-australiano), com idades de 6 anos, procedentes de plantios florestais da área experimental do Centro de Ciências Agrárias e Engenharias da Universidade Federal do Espírito Santo, munícipio de Alegre, estado do Espírito Santo.

Para determinação da densidade básica da madeira, seguiram-se as recomendações da norma ABNT NBR 7190 (1997), confeccionando-se corpos de prova nas dimensões de 2,0 x 3,0 x 5,0 cm, com a maior medida orientada no sentido longitudinal. A região de amostragem dos corpos de prova em relação à base foi entre 0 e 2,60 m e na direção medula-casca nas posições interna e intermediária. As amostras foram saturadas em um dessecador com água mediante aplicação de vácuo intermitente. Após a secagem natural, as amostras foram colocadas em uma estufa de ventilação forçada, a uma temperatura de $103^{\circ} \mathrm{C} \pm 2^{\circ} \mathrm{C}$, até atingirem massa constante, obtendo assim a sua massa seca. A densidade básica da madeira foi obtida pela razão entre a massa seca e o volume saturado. A partir das mesmas amostras foi determinada a contração da madeira tomando-se as medidas nos sentidos longitudinal, radial e tangencial na condição de madeira saturada e anidra, de acordo com a norma ABNT NBR 7190 (1997). A partir das dimensões do material nas duas condições de avaliação além das contrações lineares determinou-se a contração volumétrica e o fator anisotrópico.

\section{Ensaios destrutivos}

Para a determinação das propriedades mecânicas seguiu-se a norma ABNT NBR 7190 (1997). Foram confeccionados corpos de prova para os ensaios de compressão e cisalhamento paralelo às fibras para determinar tais resistências e o ensaio de flexão estática para determinar o módulo de elasticidade aparente paralelo às fibras $\left(M O E_{a, s t}\right)$ e o módulo de ruptura (MOR). Os ensaios mecânicos foram realizados em corpos de prova, mantidos em equilíbrio higroscópico com as condições de temperatura e umidade relativa ambiental, mediante utilização de uma máquina universal de ensaios com capacidade de $100 \mathrm{kN}$.

A madeira de cedro-australiano utilizada para determinar o módulo de elasticidade longitudinal aparente $\left(M O E_{a, s t}\right)$ no ensaio destrutivo de flexão estática para três pontos foi processada para obtenção dos corpos de prova de seção $30 \times 30 \mathrm{~mm}(b \times h)$ respeitando as proporções estabelecidas na norma ABNT NBR 7190 (1997) e testadas em máquina de ensaios com vão variável $(L)$ resultando nas seguintes relações $(L / h)$ : $23,21,19,17,14,11,8$ e 5, conforme a Figura 1 . Para cada relação de $L / h$ foram utilizadas seis repetições. Todos os corpos de prova foram armazenados em local coberto e mantidos em equilíbrio higroscópico com o meio, condição prévia para execução dos ensaios. O comprimento total dos corpos de prova é a soma do vão $(L)$ com as extremidades distantes $25 \mathrm{~mm}$ dos apoios. 


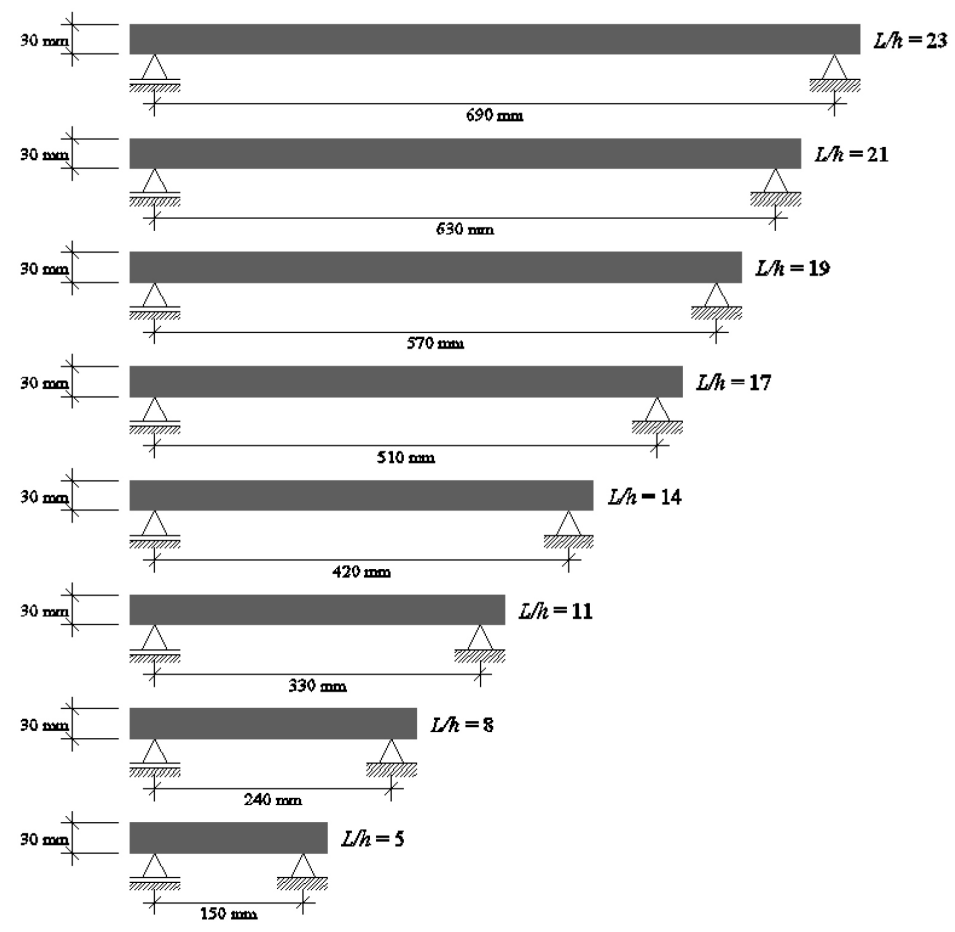

FIGURA 1: Esquema do vão e altura dos corpos de prova para os ensaios de flexão estática.

FIGURE 1: Range and height scheme of specimens for static bending.

O ensaio destrutivo de flexão estática a três pontos nos corpos de prova foi realizado para obter o módulo de elasticidade aparente $\left(M O E_{a, s t}\right)$ (Equação 1), e o módulo de ruptura (MOR) (Equação 2), conforme a norma ASTM D - 198 (2008).

$$
\begin{aligned}
& M O E_{a, s t}=\frac{P \cdot L^{3}}{4 \cdot b \cdot h^{3} \cdot \delta} \\
& \mathrm{MOR}=\frac{3 \cdot P_{r u p} \cdot L}{2 \cdot b \cdot h^{2}}
\end{aligned}
$$

Sendo $M O E_{a, s t}$ o módulo de elasticidade longitudinal aparente (MPa), MOR o módulo de ruptura (MPa), $P$ o incremento de força aplicada $(\mathrm{N}), P_{r u p}$ a força de ruptura $(\mathrm{N}), L$ a distância entre apoios $(\mathrm{mm})$, $b$ a largura $(\mathrm{mm}), h$ a altura $(\mathrm{mm}), \delta$ o deslocamento vertical devido ao incremento de força aplicada $(\mathrm{mm})$.

\section{Ensaios não destrutivos}

Os ensaios não destrutivos de vibração transversal livre e vibração longitudinal foram realizados nos mesmos corpos de prova utilizados para os ensaios destrutivos de flexão estática para três pontos.

\section{Ensaio de vibração transversal livre}

O ensaio de vibração transversal livre foi realizado conforme instruções da norma ASTM E - 1876 (2007) e ASTM C - 215 (2008). Para a realização do experimento foi utilizado um microfone para captar a vibração devido à aplicação de um impacto (Figura 2), mediante um suporte para o corpo de prova e usando um software livre (FFT analyzer) no computador, para capturar a frequência natural. Para o cálculo do módulo de elasticidade longitudinal $\left(M O E_{a, v}\right)$, aplicou-se a Equação 3. Devido à vibração transversal livre, tomou-se a primeira frequência de ressonância. 


$$
M O E_{a, v t}=\left(\frac{2 \cdot f_{1}}{\gamma_{1} \cdot \pi}\right)^{2} \cdot \frac{m \cdot l^{3}}{I}
$$

Sendo $M O E_{a, v t}$ o módulo de elasticidade longitudinal obtido pelo método de vibração transversal livre $(\mathrm{MPa}), f_{1}$ a primeira frequência de vibração transversal livre $(\mathrm{Hz}), \gamma_{1}$ obtido por meio da equação $(n+$ $0,5)^{2}$, em que $n=1$ (número do modo harmônico), $m$ a massa do corpo de prova $(\mathrm{kg}$ ), $l$ o comprimento da peça $(\mathrm{m}), I$ o momento de inércia da seção transversal $\left(\mathrm{m}^{4}\right)$.

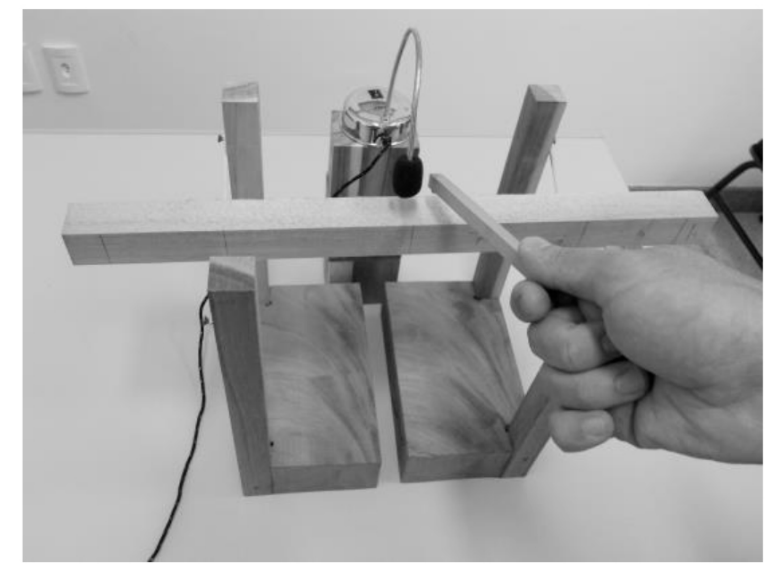

FIGURA 2: Ensaio de vibração transversal livre.

FIGURE 2: Transverse vibration testing.

\section{Ensaio de vibração longitudinal}

O ensaio de vibração longitudinal foi realizado conforme instruções da norma ASTM E - 1876 (2007), no qual a direção do movimento da onda ocorre na mesma direção do modo de vibração longitudinal. O ensaio de vibração longitudinal foi realizado de forma semelhante ao de vibração transversal livre, mas com sentido do impacto e captação do som na direção longitudinal (Figura 3). No ensaio de vibração longitudinal obtém-se uma resposta acústica composta por uma ou mais frequências naturais de vibração longitudinal. Para o cálculo do módulo de elasticidade longitudinal ( $M O E_{n}$ ) $)$ utilizou-se a Equação 4.

$M O E_{a, l}=4 \cdot\left(\frac{m \cdot f_{l}^{2}}{b}\right)^{2} \cdot\left(\frac{l}{h}\right)$

Sendo $M O E_{a, l}$ o módulo de elasticidade longitudinal obtido pelo método de vibração longitudinal (MPa), $m$ a massa (kg) do corpo de prova, $f_{l}$ a primeira frequência de vibração longitudinal (Hz), $b$ a largura $(\mathrm{mm}), h$ a altura $(\mathrm{mm}), l \mathrm{o}$ comprimento $(\mathrm{mm})$. 


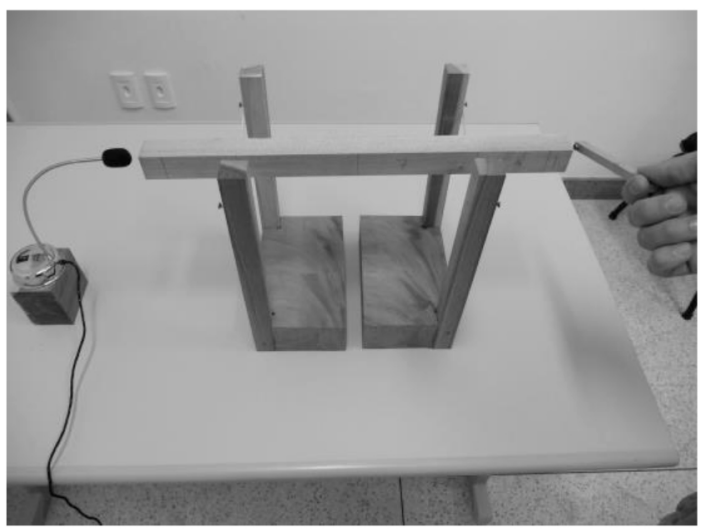

FIGURA 3: Ensaio de vibração longitudinal.

FIGURE 3: Longitudinal vibration testing.

\section{Análise dos dados}

A análise dos dados foi realizada considerando os modelos estatísticos: polinomial quadrático com resposta em platô (MPQ), não linear exponencial $1 \mathrm{com}$ resposta em platô (MNLE1) e não linear exponencial $2 \mathrm{com}$ resposta em platô (MNLE2). O ajuste dos modelos de platô de resposta foi realizado a partir dos dados obtidos experimentalmente para as oito relações $L / h$ estudadas e seis repetições por meio do procedimento NLIN (PROC NLIN) do software estatístico SAS (SAS INSTITUTE, 2004).

\section{Modelo polinomial quadrático com resposta em platô (MPQ)}

Consideraram-se os seguintes modelos:

$$
\begin{array}{llll}
Y=a+b x+c x^{2}+e & \text { se } & x<x_{0} & \text { (quadrático) } \\
Y=p+e & \text { se } & x \geq x_{0} & \text { (platô) }
\end{array}
$$

Sendo $Y$ o módulo de elasticidade longitudinal aparente $\left(E_{a}\right)$ referente à relação $L / h$. Dessa forma, para valores de $x$ menores que $x_{0}$, o modelo que descreve a resposta $Y$ é uma função quadrática, e para valores de $x$ maiores ou iguais a $x_{0}$, o modelo é uma constante ou platô. Para estimação dos parâmetros, o modelo deve apresentar propriedades matemáticas adequadas, isto é, deve ser uma função contínua e diferençável em $x_{0}$. Deriva-se a equação igualando-a a zero, sendo $x=x_{0}$. Esta condição implica que: $x_{0}=-$ $b / 2 c$ e $p=a-\left(b^{2} / 4 c\right)$; em que $x_{0}$ é a máxima relação $L / h$ para um máximo módulo de elasticidade longitudinal aparente e o ponto de intercessão das duas linhas $p$ é o platô, sendo $a, b$ e $c$ os parâmetros do modelo a serem estimados.

\section{Modelo não linear exponencial 1 com resposta em platô (MNLE1)}

Consideraram-se os seguintes modelos:

$$
\begin{array}{llll}
Y=a \cdot \exp \left[-c(x-b)^{2}\right]+e & \text { se } & x<x_{0} & \text { (exponencial) } \\
Y=p+e & \text { se } & x \geq x_{0} & \text { (platô) }
\end{array}
$$

Da mesma forma que no modelo (i), ao se derivar $Y$ em relação a $x$, tem-se:

$$
\frac{\partial}{\partial x}=\frac{\partial\left\{a \cdot \exp \left[-c(x-b)^{2}\right]\right\}}{\partial}=a \cdot \exp \left[-c(x-b)^{2}\right] \cdot[-2 c(x-b)]
$$

Igualando-se o resultado da derivação a zero e resolvendo-se para $x=x_{0}$, obtém-se $x_{0}=b$. Substituindo- 
se $x$ pelo valor de $x_{0}$ no modelo inicial, encontra-se $p=f\left(x_{0}\right)=a \cdot \exp \left[c(b-b)^{2}\right]$, que resulta em $p=a$, sendo $x_{0}$ a máxima relação $L / h$ para um módulo de elasticidade longitudinal aparente máximo $E_{a}, p$ o platô e $a, b$ e $c$ os parâmetros do modelo a serem estimados.

\section{Modelo não linear exponencial 2 com resposta em platô (MNLE2)}

Consideraram-se os seguintes modelos:

$$
\begin{array}{llll}
Y=a \cdot \exp \left(b x-c x^{2}\right)+e & \text { se } & x<x_{0} & \text { (exponencial) } \\
Y=p+e & \text { se } & x \geq x_{0} & \text { (platô) }
\end{array}
$$

Sendo $Y$ o módulo de elasticidade longitudinal aparente $\left(E_{a}\right)$ para uma relação $L / h$ e o ponto de intercessão das duas linhas; $p$ é o platô, $a, b$ e $c$ são os parâmetros do modelo a serem estimados.

Como apresentado nos modelos anteriores, a primeira derivada parcial deve ser idêntica no ponto $x=x_{0}$ para que a condição de continuidade das curvas antes e após o platô seja atendida. Assim, ao se derivar $y$ em relação a $x$, tem-se:

$$
\frac{\partial}{\partial}=\frac{\partial\left[a \cdot \exp \left(b x-b x^{2}\right)\right]}{\partial}=a \cdot(b-2 c x) \cdot \exp \left(b x-c x^{2}\right)
$$

Igualando-se o resultado da derivação a zero e resolvendo-se para $x=x_{0}$, obtém-se $x_{0}=b / 2 c$. Substituindo-se $x$ pelo valor de $x_{0}$ no modelo inicial, encontra-se $p=f\left(x_{0}\right)=a \cdot \exp \left[b^{2} / 2 c-c\left(b^{2} / 4 c^{2}\right)\right]$, que resulta em $p=a \cdot \exp \left(b^{2} / 4 c\right)$, em que $x_{0}$ é a máxima relação $L / h$ para um módulo de elasticidade longitudinal aparente máximo; $E_{a}$, o ponto de interseção das duas linhas; $p$ é o platô e $a, b$ e $c$ são parâmetros do modelo a serem estimados.

\section{Avaliação dos modelos de platô}

Os modelos utilizados foram avaliados por meio do coeficiente de determinação $\left(R^{2}\right)$ calculado pela expressão, sendo o quadrado da correlação entre as médias dos tratamentos observados e as preditas pelo modelo de platô, e erro padrão relativo que avalia a precisão.

$$
S_{y x}=\sqrt{\frac{\sum_{i=1}^{n}(Y-\hat{Y})^{2}}{n-p}} \quad \text { e } \quad S_{y x}(\%)=\frac{S_{y x}}{\bar{Y}} \cdot 100
$$

Sendo o erro padrão residual (MPa), o erro padrão relativo (\%), o valor estimado pelo modelo, o valor observado, a média de, o número de observações, o número de parâmetros.

\section{RESULTADOS E DISCUSSÃO}

Os valores médios da densidade básica da madeira e as contrações tangencial, radial, volumétrica e o fator anisotrópico para madeira de cedro-australiano de 6 anos de idade são apresentados na Tabela 1.

TABELA 1: Valores médios da densidade básica e os coeficientes de retratilidade da madeira de cedro-australiano tomados aos 6 anos de idade.

TABLE 1: Average values of the specific gravity and the shrinkage coefficient of Australian red cedar wood at 6 years old age.

\begin{tabular}{ccccc}
\hline$p_{\text {bas }}\left(\mathrm{g} / \mathrm{m}^{3}\right)$ & $\mathrm{CT}(\%)$ & $\mathrm{CR}(\%)$ & $\mathrm{CV}(\%)$ & $\mathrm{FA}(\mathrm{CT} / \mathrm{CR})$ \\
\hline $0,277(0,045)$ & $8,54(2,14)$ & $3,84(1,51)$ & $12,07(1,71)$ & $2,60(1,14)$ \\
\hline
\end{tabular}

Em que: Valores entre parênteses se referem ao desvio padrão; $p_{\text {bas }}=$ Densidade básica; $\mathrm{CT}=$ Contração tangencial; $\mathrm{CR}=$ Contração radial; $\mathrm{CV}=$ Contração volumétrica; $\mathrm{FA}=$ Fator anisotrópico. 
O valor médio de densidade básica da madeira de cedro-australiano apresentado na Tabela 1 é compatível com os valores médios apresentados por Braz et al. (2013) para madeira de cedro-australiano de 6 anos de idade que foi igual a $0,293 \mathrm{~g} / \mathrm{m}^{3}$, Nassur (2010), para madeira de cedro-australiano de 18 anos de idade que foi igual a $0,314 \mathrm{~g} / \mathrm{m}^{3}$ e Ziech (2008) para madeira de cedro-australiano de 4 anos de idade que foi igual a $0,306 \mathrm{~g} / \mathrm{m}^{3}$. De acordo com a classificação da densidade básica da madeira proposta pelo Forest Products Laboratory (1973), tal madeira de cedro-australiano selecionada para a pesquisa pode ser classificada como madeira leve.

Na Tabela 2 estão os valores médios para a resistência característica à compressão paralela às fibras $\left(f_{c 0, k}\right)$, resistência característica ao cisalhamento paralelo às fibras $\left(f_{v 0, k}\right)$, módulo de elasticidade médio à compressão paralela às fibras $\left(E_{c 0, m}\right)$ da madeira de cedro-australiano, caracterizados segundo a ABNT NBR 7190 (1997) e corrigidos para a umidade de referência igual a $12 \%$, pois encontravam-se em umidade de equilíbrio higroscópico com o meio que foi igual a 13,04\% com coeficiente de variação igual a 5,22\%.

TABELA 2: Valores médios da resistência característica à compressão paralela às fibras, cisalhamento paralelo às fibras da madeira e módulo de elasticidade médio à compressão paralela às fibras da madeira de cedroaustraliano.

TABLE 2: Mean values of characteristic resistance to compression parallel to grain, characteristic shear strength parallel to wood fiber and medium compression elasticity modulus, parallel to wood fibers of Australian red cedar.

\begin{tabular}{ccc}
\hline$f_{c 0, k}(\mathrm{MPa})$ & $f_{v 0, k}(\mathrm{MPa})$ & $E_{c 0, m}(\mathrm{MPa})$ \\
\hline 23,33 & 6,54 & 6975,13 \\
\hline
\end{tabular}

Em que: $f_{\mathrm{c} 0, \mathrm{k}}=$ Resistência característica à compressão paralela às fibras da madeira; $f_{\mathrm{v} 0, \mathrm{k}}=$ Resistência característica ao cisalhamento paralelo às fibras da madeira; $E_{\mathrm{c} 0, \mathrm{~m}}=$ Módulo de elasticidade médio à compressão paralela às fibras da madeira.

A madeira de cedro-australiano pode ser classificada na classe de resistência C20 das dicotiledôneas (folhosa) com base nas propriedades mecânicas apresentadas na Tabela 2 que define valores mínimos de resistência característica à compressão paralela às fibras $\left(f_{c 0, k}\right)$ igual a $20 \mathrm{MPa}$, resistência característica ao cisalhamento paralelo às fibras $\left(f_{v 0, k}\right)$ igual a $4 \mathrm{MPa}$, módulo de elasticidade médio à compressão paralela às fibras $\left(E_{c 0 . m}\right)$ de $3500 \mathrm{MPa}$, densidade básica média igual a $400 \mathrm{~kg} \mathrm{~m}^{-3}$, densidade aparente igual a $500 \mathrm{~kg}$ $\mathrm{m}^{-3}$, umidade de referência igual a 12\%, segundo a ABNT NBR 7190 (1997).

$\mathrm{Na}$ Tabela 3 estão os valores médios do módulo de elasticidade longitudinal aparente $\left(M O E_{a, s t}\right)$ e módulo de ruptura (MOR) obtidos por meio dos ensaios de flexão estática, módulo de elasticidade longitudinal aparente obtidos pelo ensaio de vibração transversal livre $\left(M O E_{a, v}\right)$ e módulo de elasticidade longitudinal aparente obtidos pelo ensaio de vibração longitudinal $\left(M O E_{a, l}\right)$. Todos os ensaios tiveram a mesma relação $L / h$ para madeira de cedro-australiano e a umidade média dos corpos de prova mantidos em equilíbrio higroscópico com o meio, armazenados em local coberto, foi igual a 13,04\%, com coeficiente de variação igual a 5,22\%. 
TABELA 3: Valores médios do módulo de elasticidade longitudinal aparente e módulo de ruptura para cada relação $\mathrm{L} / \mathrm{h}$ para madeira de cedro-australiano.

TABLE 3: Mean values of the modulus of apparent longitudinal elasticity and modulus of rupture for each $\mathrm{L} / \mathrm{h}$ in Australian red cedar wood.

\begin{tabular}{ccccc}
\hline \multirow{2}{*}{$L / h$} & \multicolumn{3}{c}{ Módulo de elasticidade longitudinal aparente } & \multirow{2}{*}{ MOR (MPa) } \\
\cline { 2 - 4 } & $M O E_{a, s t}(\mathrm{MPa})$ & $M O E_{a, v t}(\mathrm{MPa})$ & $M O E_{a, l}(\mathrm{MPa})$ & \\
\hline 5 & 1228,73 & 5436,55 & 6931,08 & 33,49 \\
8 & 3131,87 & 5507,10 & 6663,17 & 36,70 \\
11 & 4682,28 & 6363,96 & 7015,19 & 43,81 \\
14 & 5642,45 & 6657,25 & 7170,60 & 44,72 \\
17 & 6162,92 & 6946,36 & 7371,80 & 44,03 \\
19 & 6084,32 & 6915,21 & 7312,18 & 44,64 \\
21 & 6363,28 & 6788,16 & 7267,61 & 43,67 \\
23 & 6307,28 & 6790,46 & 7125,06 & 43,75 \\
\hline
\end{tabular}

Em que: $M O E_{\text {a.st }}=$ Módulo de elasticidade longitudinal aparente obtido pelo ensaio de flexão estática; $M O R=$ Módulo de ruptura obtido pelo ensaio de flexão estática; $M O E_{\text {a,vt }}=$ Módulo de elasticidade longitudinal aparente obtido pelo ensaio de vibração transversal livre; $M O E_{\mathrm{a}, 1}=$ Módulo de elasticidade longitudinal aparente obtido pelo ensaio de vibração longitudinal.

Na Tabela 4 está o teste F para falta de ajustamento, também chamado The Lack of Fit F-test, para os três modelos estatísticos ajustados para madeira de cedro-australiano com a carga aplicada, formando ângulos quaisquer com as direções tangencial e radial, no ensaio de flexão estática.

De acordo com a Tabela 4, a falta de ajustamento foi não significativa para os três modelos a $5 \%$ de probabilidade $(\mathrm{P}>0,05)$ para o ensaio de flexão estática.

$\mathrm{Na}$ Tabela 5 estão as equações ajustadas para os modelos de regressão utilizados para madeira de cedro-australiano no ensaio de flexão estática, com os coeficientes de determinação $R^{2}$, relação máxima $x_{0}$ e platô $p$.

TABELA 4: Análise de variância e teste F para falta de ajustamento para os modelos analisados para madeira de cedro-australiano com a carga aplicada formando ângulos quaisquer com as direções tangencial e radial no ensaio de flexão estática.

TABLE 4: Analysis of variance and $\mathrm{F}$ test, for the lack of adjustment for the analyzed models of Australian red cedar wood, with applied load forming varied angles with the tangential and radial directions in static bending test.

\begin{tabular}{cccccc}
\hline Modelos & Fonte de Variação & G. L. & Q. M. & F & Valor-P \\
\hline \multirow{4}{*}{ MPQ } & Mod. Reg. não Cor. & 3 & 440613104,40 & & \\
& Falta de Ajust. & 5 & 55763,28 & $0,397^{\text {ns }}$ & 0,848 \\
& Resíduo (Erro Puro) & 40 & 140586,30 & & \\
\hline \multirow{5}{*}{ MNLE1 } & Mod. Reg. não Cor. & 3 & 440220000,00 & \\
& Falta de Ajust. & 5 & 293751,60 & $2,089^{\text {ns }}$ & 0,087 \\
& Resíduo (Erro Puro) & 40 & 140586,30 & \\
\hline \multirow{5}{*}{ MNLE2 } & Mod. Reg. não Cor. & 3 & 440220000,00 & \\
& Falta de Ajust & 5 & 293751,60 & $2,089^{\text {ns }}$ & 0,087 \\
& Resíduo (Erro Puro) & 40 & 140586,30 & \\
\hline
\end{tabular}

Em que: ${ }^{\text {ns }}=$ Não significativo $(\mathrm{P}>0,05) ; \mathrm{G} . \mathrm{L} .=$ Graus de liberdade; $\mathrm{Q} . \mathrm{M} .=$ Quadrado médio; $\mathrm{F}=$ Análise de variância; Valor-P = Probabilidade; $\mathrm{MPQ}=$ Modelo polinomial quadrático com resposta em platô; MNLE1 = Modelo não linear exponencial 1 com resposta em platô; MNLE2 = Modelo não linear exponencial 2 com resposta em platô. 
TABELA 5: Equações ajustadas para os diferentes modelos, coeficiente de determinação, relação máxima L/h e platô para madeira de cedro-australiano com a carga aplicada formando ângulos quaisquer com as direções tangencial e radial no ensaio de flexão estática.

TABLE 5: Equations adjusted for the different models, coefficient of determination, maximum ratio $\mathrm{L} / \mathrm{h}$ and plateau for Australian red cedar, with applied load forming varied angles with the tangential and radial directions in static bending test.

\begin{tabular}{clcccc}
\hline Modelos & \multicolumn{1}{c}{ Equações ajustadas } & $R^{2}(\%)$ & $S_{y x}(\%)$ & $x_{0}(L / h)$ & $p(\mathrm{MPa})$ \\
\hline MPQ & $\hat{y}=-3107,6+997,4 x-26,5742 x^{2}$ & 99,81 & 1,78 & 18,77 & 6251,11 \\
\hline MNLE1 & $\hat{y}=6195,7 \exp \left[-0,0117(x-16,0516)^{2}\right]$ & 99,01 & 4,08 & 16,05 & 6195,70 \\
\hline MNLE2 & $\hat{y}=304,6 \exp \left(0,3753 x-0,0117 x^{2}\right)$ & 99,03 & 4,04 & 16,05 & 6195,70 \\
\hline
\end{tabular}

Em que: " ns $=$ Não significativo $(\mathrm{P}>0,05) ; R^{2}(\%)=$ Coeficiente de determinação; $S_{y x}(\%)=$ Erro padrão relativo; $x_{0}(L / h)$ $=$ Ponto de interseção das duas linhas do modelo no ponto máximo; $p(\mathrm{MPa})=$ Platô do modelo; $\mathrm{MPQ}=$ Modelo polinomial quadrático com resposta em platô; MNLE1 = Modelo não linear exponencial 1 com resposta em platô; MNLE2 $=$ Modelo não linear exponencial 2 com resposta em platô.

Analisando os resultados da Tabela 5, pode-se notar que os coeficientes de determinação para todos os modelos mostraram-se altos, com valores semelhantes, e erro padrão relativo com valores inferiores a $4,08 \%$. Verifica-se, também, que os modelos MNLE1 e MNLE2 obtiveram valores idênticos para $x_{0}(L / h)$ e $p(\mathrm{MPa})$ no ensaio de flexão estática. No entanto, o modelo MPQ obteve os maiores valores para a relação máxima $\left(x_{0}\right)$ e platô $(p)$, em comparação com os demais modelos.

$\mathrm{Na}$ Tabela 6 está o teste $\mathrm{F}$ para falta de ajustamento para os três modelos ajustados, para madeira de cedro-australiano no ensaio de vibração transversal livre, formando ângulos quaisquer com as direções tangencial e radial.

TABELA 6: Análise de variância e teste F para falta de ajustamento para os modelos analisados, para madeira de cedro-australiano no ensaio de vibração transversal livre, formando ângulos quaisquer com as direções tangencial e radial.

TABLE 6: Analysis of variance and F test, with lack of adjustment for the models analyzed, on Australian red cedar wood free transverse vibration testing, forming varied angles with the tangential and radial directions.

\begin{tabular}{cccccc}
\hline Modelos & Fonte de Variação & G. L. & Q. M. & F & Valor-P \\
\hline \multirow{4}{*}{ MPQ } & Mod. Reg. não Cor. & 3 & 665560045,40 & & \\
& Falta de Ajustamento & 5 & 221613,60 & $0,755^{\text {ns }}$ & 0,588 \\
& Resíduo (Erro Puro) & 40 & 293472,65 & & \\
\hline \multirow{4}{*}{ MNLE1 1} & Mod. Reg. não Cor. & 3 & 665600000,00 & \\
& Falta de Ajustamento & 5 & 197572,80 & $0,673^{\text {ns }}$ & 0,646 \\
& Resíduo (Erro Puro) & 40 & 293472,65 & & \\
\hline \multirow{4}{*}{ MNLE2 } & Mod. Reg. não Cor. & 3 & 665600000,00 & & \\
& Falta de Ajustamento & 5 & 197572,80 & $0673^{\text {ns }}$ & 0,646 \\
& Resíduo (Erro Puro) & 40 & 293472,65 & & \\
\hline
\end{tabular}

Em que: ${ }^{\text {ns }}=$ Não significativo $(\mathrm{P}>0,05) ; \mathrm{G} . \mathrm{L} .=$ Graus de liberdade; $\mathrm{Q} . \mathrm{M} .=$ Quadrado médio; $\mathrm{F}=$ Análise de variância; Valor-P = Probabilidade; $\mathrm{MPQ}=$ Modelo polinomial quadrático com resposta em platô; $\mathrm{MNLE} 1=$ Modelo não linear exponencial 1 com resposta em platô; MNLE2 = Modelo não linear exponencial 2 com resposta em platô.

De acordo com a Tabela 6 , a falta de ajustamento foi não significativa para os três modelos a $5 \%$ de 
probabilidade $(\mathrm{P}>0,05)$ para o ensaio de vibração transversal livre.

$\mathrm{Na}$ Tabela 6 estão apresentadas as equações ajustadas para os modelos de platô para madeira de cedro-australiano, no ensaio de vibração transversal livre, formando ângulos quaisquer com as direções tangencial e radial, com os coeficientes de determinação $R^{2}$, relação máxima $x_{0}$ e platô $p$.

TABELA 7: Equações ajustadas para os diferentes modelos, coeficiente de determinação, relação máxima L/h e platô para madeira de cedro-australiano para o ensaio de vibração transversal livre formando ângulos quaisquer com as direções tangencial e radial.

TABLE 7: Equations adjusted for the different models, coefficient of determination, maximum ratio $\mathrm{L} / \mathrm{h}$, and plateau on Australian red cedar wood for free transverse vibration testing, forming varied angles with the tangential and radial directions.

\begin{tabular}{clcccc}
\hline Modelos & Equações ajustadas & $R^{2}(\%)$ & $S_{y x}(\%)$ & $x_{0}(L / h)$ & $p(\mathrm{MPa})$ \\
\hline MPQ & $\hat{y}=4007,5+286,8 x-7,1914 x^{2}$ & 93,05 & 2,73 & 19,94 & 6866,46 \\
\hline MNLE1 & $\hat{y}=6865,5 \exp \left[-0,00131(x-19,2533)^{2}\right]$ & 93,80 & 2,58 & 19,25 & 6865,55 \\
\hline MNLE2 & $\hat{y}=4230,2 \exp \left(0,0503 x-0,00131 x^{2}\right)$ & 93,68 & 2,60 & 19,25 & 6865,55 \\
\hline
\end{tabular}

Em que: ${ }^{\text {ns }}=$ Não significativo $(\mathrm{P}>0,05) ; R^{2}(\%)=$ Coeficiente de determinação; $S_{y x}(\%)=$ Erro padrão relativo; $x_{0}(L / h)$ $=$ Ponto de interseção das duas linhas do modelo no ponto máximo; $p(\mathrm{MPa})=$ Platô do modelo; $\mathrm{MPQ}=$ Modelo polinomial quadrático com resposta em platô; MNLE1 = Modelo não linear exponencial 1 com resposta em platô; MNLE2 = Modelo não linear exponencial 2 com resposta em platô.

Analisando a Tabela 7, pode-se notar que os modelos tiveram comportamento idêntico aos da Tabela 5. Os modelos MNLE1 e MNLE2 obtiveram valores iguais para $x_{0}(L / h)$ e de $p(\mathrm{MPa})$ no ensaio de vibração transversal livre, sendo todos os modelos com valores de $R^{2}$ próximos e erro padrão relativo com valores inferiores a 2,73\%; o modelo MPQ obteve os maiores valores para a relação máxima $\left(x_{0}\right)$ e platô $(p)$, em comparação com os demais modelos.

A equação de regressão linear ajustada para o módulo de elasticidade longitudinal aparente $E_{a, l} \mathrm{em}$ função da relação $L / h$ para o ensaio de vibração longitudinal, juntamente com o respectivo coeficiente de determinação $\left(R^{2}\right)$ e erro padrão relativo $\left(S_{y x}\right)$ é mostrada na Tabela 8. Pode-se notar que o coeficiente de regressão foi não significativo ( $\mathrm{P}>0,05)$, explicando somente $0,95 \%$ de variação observada para o módulo de elasticidade longitudinal aparente, e que o interceptor foi significativo $(\mathrm{P}<0,05)$ com este modelo, apresentando erro padrão relativo igual a $3,11 \%$. Pela Figura $4 \mathrm{c}$ a equação representativa seria uma reta paralela ao eixo $L / h$, isto é, .

TABELA 8: Equação de regressão linear ajustada.

TABLE 8: Adjusted linear regression equation .

\begin{tabular}{cccccc}
\hline$\hat{y}(\mathrm{MPa})$ & $x$ & $a(\mathrm{MPa})$ & $b(\mathrm{MPa})$ & $R^{2}(\%)$ & $S_{y x}(\%)$ \\
\hline$E_{a, l}$ & $L / h$ & $9,532^{\mathrm{ns}}$ & $7031,283^{* *}$ & $0,95^{\mathrm{ns}}$ & 3,11 \\
\hline
\end{tabular}

Em que: ${ }^{\text {ns }}=$ Não significativo $(\mathrm{P}>0,05) ; * *=$ Significativo $(\mathrm{P}<0,05) ; \hat{y}(\mathrm{MPa})=$ Equação ajustada; $x=$ Relação $L / h$; $a(\mathrm{MPa})=$ Coeficiente de regressão; $b(\mathrm{MPa})=$ Interceptor; $R^{2}(\%)=$ Coeficiente de determinação; $S_{v x}(\%)=$ Erro padrão relativo.

Na Figura 4 são apresentadas e comparadas graficamente as curvas experimentais e teóricas dos três modelos de regressão para o módulo de elasticidade longitudinal aparente, em função da relação $L / h$ para 
madeira de cedro-australiano, com a carga aplicada formando ângulos quaisquer com as direções tangencial e radial nos ensaios de flexão estática, vibração transversal livre e vibração longitudinal.
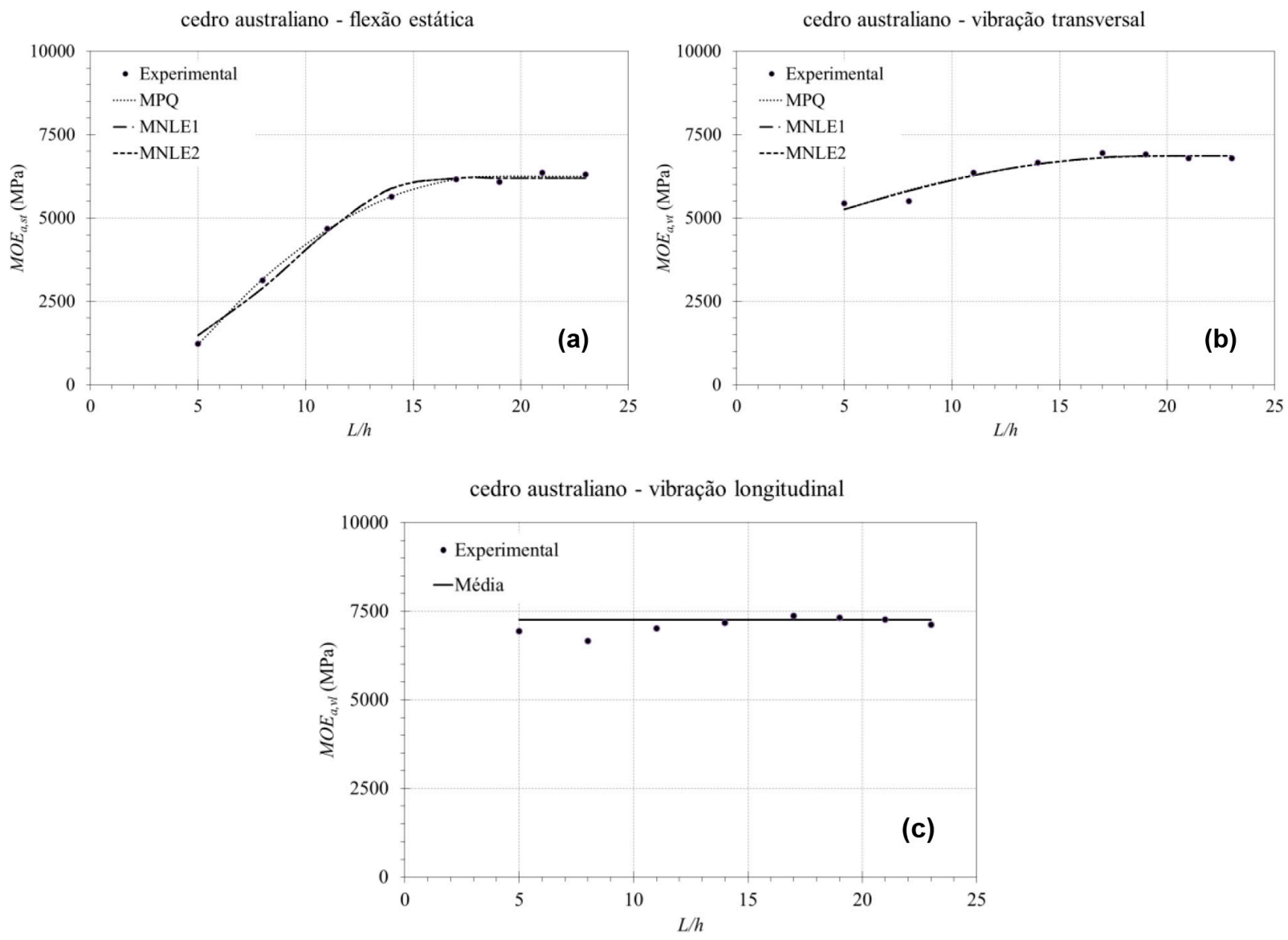

FIGURA 4: Curvas do módulo de elasticidade aparente em função da relação $\mathrm{L} / \mathrm{h}$ para madeira de cedro-australiano, referente à carga aplicada, formando ângulos quaisquer com as direções tangencial e radial nos ensaios de flexão estática (a), vibração transversal livre (b) e vibração longitudinal (c).

FIGURE 4: Curves of apparent modulus of elasticity versus the ratio $\mathrm{L} / \mathrm{h}$ for Australian red cedar wood, referring to applied load, forming varied angles with the tangential and radial directions, in testings of static bending (a), free transverse vibration (b) and vibration longitudinal (c).

A curva do módulo de elasticidade aparente $M O E_{a, s t}$ obtido no ensaio de flexão estática a três pontos demonstra a comparação dos modelos estatísticos em um único gráfico, facilitando a visualização da assíntota horizontal (Figura 4a). Isto significa que, a partir do início da assíntota horizontal, a influência do cisalhamento é mínima, de modo que pode ser considerada insignificante. Além disso, o gráfico com curva acentuada apresenta a queda da eficácia do teste de flexão estática quando se deseja obter a elasticidade da madeira em peças com razão $L / h$ menor do que a recomendável. Seguindo a mesma linha de raciocínio, a Figura $5 \mathrm{~b}$ ilustra a curva do módulo de elasticidade aparente $E_{a, v t}$ obtido no ensaio de vibração transversal livre, sendo possível visualizar a comparação dos modelos estatísticos no gráfico. Percebe-se que, neste tipo de ensaio, a influência das tensões cisalhantes diminui. O cisalhamento não influencia, ou é insignificante, no módulo de elasticidade da peça após o ponto em que a linha tende a ser uma reta. Na Figura 5c, que trata da vibração longitudinal, observa-se analiticamente que não há inclinação; logo, é uma linha reta, e isso ocorre devido ao grau de precisão do teste não destrutivo de vibração longitudinal. Dessa forma, os resultados do módulo de elasticidade longitudinal aparente $E_{a, l}$ não se alteram devido às tensões cisalhantes com a variação da relação $L / h$.

Na Tabela 9 está o teste F para falta de ajustamento para os três modelos estatísticos ajustados para 
madeira de cedro-australiano, com a carga aplicada formando ângulos quaisquer com as direções tangencial e radial no ensaio de flexão estática.

TABELA 9: Análise de variância e teste F para falta de ajustamento para os três modelos estatísticos ajustados para madeira de cedro-australiano, com a carga aplicada formando ângulos quaisquer com as direções tangencial e radial no ensaio de flexão estática.

TABLE 9: Analysis of variance and $\mathrm{F}$ test on the lack of adjustment for the models analyzed in Australian rd cedar wood, with applied load forming varied angles with the tangential and radial directions in static bending test.

\begin{tabular}{cccccc}
\hline Modelos & Fonte de Variação & G. L. & Q. M. & F & Valor-P \\
\hline \multirow{4}{*}{ MPQ } & Mod. Reg. não Cor. & 3 & 28265,834 & & \\
& Falta de Ajust. & 5 & 8,759 & $0,3945^{\text {ns }}$ & 0 \\
& Resíduo (Erro Puro) & 40 & 22,202 & & \\
\hline \multirow{4}{*}{ MNLE1 } & Mod. Reg. não Cor. & 3 & 28267,600 & & \\
& Falta de Ajust. & 5 & 7,645 & $0,3443^{\text {ns }}$ & 0,883 \\
& Resíduo (Erro Puro) & 40 & 22,202 & & \\
\hline \multirow{4}{*}{ MNLE2 } & Mod. Reg. não Cor. & 3 & 28267,600 & & \\
& Falta de Ajust & 5 & 7,645 & $0,3443^{\text {ns }}$ & 0,883 \\
& Resíduo (Erro Puro) & 40 & 22,202 & & \\
\hline
\end{tabular}

Em que: ${ }^{\text {ns }}=$ Não significativo $(\mathrm{P}>0,05) ; G . L .=$ Graus de liberdade; $\mathrm{Q} . \mathrm{M} .=$ Quadrado médio; $\mathrm{F}=$ Análise de variância; Valor-P $=$ Probabilidade; $\mathrm{MPQ}=$ Modelo polinomial quadrático com resposta em platô; $\mathrm{MNLE} 1=$ Modelo não linear exponencial 1 com resposta em platô; MNLE2 = Modelo não linear exponencial 2 com resposta em platô.

De acordo com a Tabela 9, a falta de ajustamento foi não significativa para os três modelos a 5\% probabilidade $(\mathrm{P}>0,05)$, para o ensaio de flexão estática.

$\mathrm{Na}$ Tabela 10 estão as equações ajustadas para os diferentes modelos de regressão para o ensaio de flexão estática, com os coeficientes de determinação $R^{2}$, relação máxima $x_{0}$ e platô $p$, na madeira de cedroaustraliano.

TABELA 10: Equações ajustadas para os diferentes modelos, coeficiente de determinação, relação máxima L/h e platô, para madeira de cedro-australiano com a carga aplicada formando ângulos quaisquer com as direções tangencial e radial no ensaio de flexão estática.

TABLE 10: Equations adjusted for the different models, coefficient of determination, maximum $\mathrm{L} / \mathrm{h}$ ratio, and plateau, for Australian red cedar wood with applied load forming varied angles with the tangential and radial directions in static bending test.

\begin{tabular}{cccccc}
\hline Modelos & Equações ajustadas & $R^{2}(\%)$ & $S_{y x}(\%)$ & $x_{0}(L / h)$ & $p(\mathrm{MPa})$ \\
\hline MPQ & $\hat{y}=18,5468+3,4168 x-0,1136 x^{2}$ & 94,31 & 2,63 & 15,03 & 44,23 \\
\hline MNLE1 & $\hat{y}=44,2312 \exp \left[-0,00332(x-14,5014)^{2}\right]$ & 95,02 & 2,46 & 14,50 & 44,23 \\
\hline MNLE2 & $\hat{y}=22,0005 \exp \left(0,0963 x-0,00332 x^{2}\right)$ & 95,02 & 2,46 & 14,50 & 44,23 \\
\hline
\end{tabular}

Em que: ${ }^{\text {ns }}=$ Não significativo $(\mathrm{P}>0,05) ; R^{2}(\%)=$ Coeficiente de determinação; $S_{y x}(\%)=$ Erro padrão relativo; $x_{0}(L / h)$ $=$ Ponto de interseção das duas linhas do modelo no ponto máximo; $p(\mathrm{MPa})=$ Platô do modelo; $\mathrm{MPQ}=$ Modelo polinomial quadrático com resposta em platô; MNLE1 = Modelo não linear exponencial 1 com resposta em platô; MNLE2 = Modelo não linear exponencial 2 com resposta em platô. 
Analisando a Tabela 10, pode-se notar que os modelos MPQ, MNLE1 e MNLE2 obtiveram valores próximos de $R^{2}$ e $x_{0}(L / h)$, e iguais de $p(\mathrm{MPa})$ no ensaio de flexão estática.

Na Figura 5 são apresentadas e comparadas graficamente as curvas mostrando os dados experimentais e preditas pelas três equações de regressão ajustadas para o módulo de ruptura em função da relação $L / h$ para madeira de cedro-australiano, com a carga aplicada formando ângulos quaisquer com as direções tangencial e radial no ensaio de flexão estática.

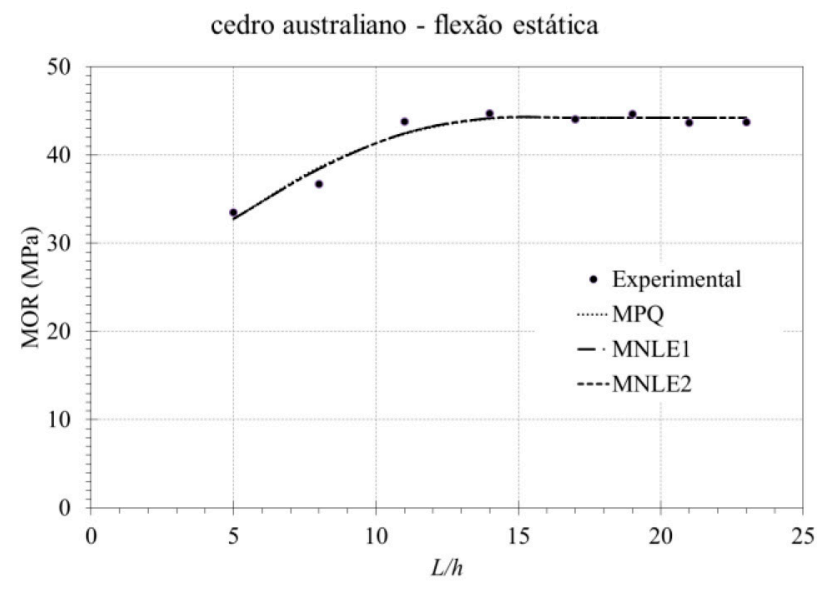

FIGURA 5: Curvas do módulo de ruptura em função da relação L/h para madeira de cedro-australiano, referente à carga aplicada formando ângulos quaisquer com as direções tangencial e radial no ensaio de flexão estática.

FIGURE 5: Curves of modulus of rupture versus $\mathrm{L} / \mathrm{h}$ ratio, for Australian red cedar wood, referring to the applied load forming varied angles with the tangential and radial directions in static bending test.

Na Figura 5 é mostrada a curva do módulo de ruptura obtido no ensaio de flexão estática a três pontos, que demonstra a comparação dos modelos estatísticos em um único gráfico, facilitando a visualização do ponto em que a linha deixa de ser curva e tende a ser reta. Isto significa que, a partir desse ponto, a influência do cisalhamento é mínima, sendo considerada insignificante. O teste mostra que os resultados do módulo de ruptura são alterados pelas tensões cisalhantes devido à variação da relação $L / h$.

Os resultados obtidos a partir desse trabalho proporcionaram condições para o aperfeiçoamento dos projetos de estruturas de madeira, cujos elementos estejam submetidos à solicitação de flexão. A relação $L / h$ igual a 21, utilizada atualmente na norma brasileira de projetos de estruturas de madeira ABNT NBR 7190 (1997) para determinar a rigidez e resistência das propriedades mecânicas de peças de madeira solicitadas à flexão, atende com segurança aos projetos.

\section{CONCLUSÕES}

A partir dessa pesquisa, constatou-se que o valor de $L / h$ igual a 21 da ABNT NBR 7190 (1997), utilizado atualmente, pode ser reduzido para $L / h$ igual a 20 , sem comprometer a segurança, tanto para determinar o módulo de elasticidade aparente $\left(M O E_{a}\right)$ quanto para determinar o módulo de ruptura (MOR). $O$ valor da relação $L / h$ igual a 16 é suficiente para não comprometer a segurança quando se trata da obtenção do MOR.

Todos os modelos avaliados mostraram-se adequados ao estudar a relação $L / h$ versus $M O E_{a}$ e $L / h$ versus MOR, tanto no ensaio de flexão estática, quanto no ensaio de vibração transversal livre, não sendo possível indicar aquele de maior qualidade. O modelo não linear exponencial $1 \mathrm{com}$ resposta em platô 
(MNLE1) pode ser recomendado, porque permite a interpretação de propriedades físicas da madeira a partir da identificação dos parâmetros do modelo.

A partir dos dados, concluiu-se que o ensaio de vibração longitudinal é o método mais adequado para obtenção do módulo de elasticidade aparente $M O E_{a, l}$ pois os esforços cortantes, devidos às tensões cisalhantes, não influenciaram significativamente com a variação da relação $L / h$.

\section{AGRADECIMENTOS}

Os autores agradecem pelo apoio do Programa Institucional de Iniciação Científica (PIIC) da Universidade Federal do Espírito Santo (UFES), ao Conselho Nacional de Desenvolvimento Científico e Tecnológico $(\mathrm{CNPq})$, pela concessão da bolsa de iniciação científica, e à Fundação de Amparo à Pesquisa do Estado do Espírito Santo (FAPES) pelo apoio aos pesquisadores por meio de projetos de pesquisa.

\section{REFERÊNCIAS}

AMERICAN SOCIETY FOR TESTING AND MATERIALS. ASTM C - 215: standard test method for fundamental transverse, longitudinal and torsional frequencies of concrete specimens. Annual Book of ASTM Standards, Philadelphia, 2008. 7 p.

AMERICAN SOCIETY FOR TESTING AND MATERIALS. ASTM D - 198: standard test methods of static tests of lumber in structural sizes. Annual Book of ASTM Standards, Philadelphia, 2008. 20 p.

AMERICAN SOCIETY FOR TESTING AND MATERIALS. ASTM E - 1876: standard test method for dynamic Young's modulus, shear modulus and Poisson's ratio by impulse excitation of vibration. Annual Book of ASTM Standards, Philadelphia, 2007. 6 p.

ASSOCIAÇÃO BRASILEIRA DE NORMAS TÉCNICAS. NBR 7190: projeto de estruturas de madeira. Rio de Janeiro, 1997. 107 p.

BRAZ, R. L. et al. Propriedades físicas e mecânicas da madeira de Toona ciliata em diferentes idades. Floresta, Curitiba, v. 43, p. 663-670, 2013.

CARREIRA, M. R.; DIAS, A. A. Classificação visual de coníferas: análise da aplicação do método norteamericano às espécies de Pinus sp plantadas no Brasil. Scientia Forestalis, Piracicaba, n. 67, p. 78-87, 2005.

CARREIRA, M. R. et al. Bending stiffness evaluation of Teca and Guajará lumber through tests of transverse and longitudinal vibration. Acta Scientiarum. Technology, Maringá, v. 34, n. 1, p. 27-32, jan./mar. 2012. FIORELLI, J.; DIAS, A. A.; COIADO, B. Propriedades mecânicas de peças com dimensões estruturais de Pinus sp: correlação entre resistência à tração e classificação visual. Revista Árvore, Viçosa, MG, v. 33, n. 4, p. 741-750, 2009.

FOREST PRODUCTS LABORATORY. FPL: standard terms for describing wood. Forest Products Laboratory Research Paper, Madison, v. 171, p. 1 - 10, 1973.

MARQUES, L. E. M. M. O papel da madeira na sustentabilidade da construção. 2008. 111 f. Dissertação (Mestrado Integrado em Engenharia Civil) - Departamento de Engenharia Civil, Faculdade de Engenharia da Universidade do Porto, Porto, 2008.

NASSUR, O. A. C. Variabilidade das propriedades tecnológicas da madeira de Toona ciliata M. Roem. com dezoito anos de idade. 2010. 84 f. Dissertação (Mestrado em Ciência e Tecnologia da Madeira) Universidade Federal de Lavras, Lavras, 2010.

SAS INSTITUTE. SAS/STAT. Version 9.1, User's Guide. USA: Cary, 2004.

SEGUNDINHO, P. G. et al. Aplicação do método de ensaio das frequências naturais de vibração para obtenção do módulo de elasticidade de peças estruturais de madeira. Revista Árvore, Viçosa, MG, v. 36, n. 6, p. 1155-1161, 2012.

TARGA, L. A.; BALLARIN, A. W.; BIAGGIONI, M. A. M. Avaliação do módulo de elasticidade da madeira com uso de método não-destrutivo de vibração transversal. Engenharia Agrícola, Jaboticabal, v. 25, n. 2, p. 291-299, 2005. 
ZIECH, R. Q. S. Estudo das características tecnológicas da madeira de cedro australiano (Toona ciliata M. Roem.). 74 f. Dissertação (Mestrado em Ciência e Tecnologia da Madeira) - Universidade Federal de Lavras, Lavras, 2008. 